\title{
TÁC ĐộNG CỦA GIÁ TRI QUẢNG CÁO ĐẾN NHậN THỨC VÀ HÀNH VI TRÁNH NÉ QUẢNG CÁO - MộT NGHIÊN CỨU VỀ TRÁNH NÉ QUẢNG CÁO TRÊN FACEBOOK TẠI TP. HỒ CHÍ MINH
}

\author{
HỒ TRÚC VI, PHAN TRỌNG NHÂN \\ Khoa Quản trị Kinh doanh, Truòng Đại học Công Nghiệp Thành Phố Hồ Chí Minh; \\ hotrucvi@iuh.edu.vn,phantrongnhan@iuh.edu.vn
}

Tóm tắt. Nghiên cứu này nhằm khám phá và khẳng định các yếu tố tác động đến nhận thức và hành vi
tránh né quảng cáo trong môi trường mạng xã hội Facebook. Dữ liệu được thu thập từ 250 khách hàng đang
sinh sống và làm việc tại khu vực TPHCM. Mô hình nghiên cứu và các thang đo được xây dựng từ các cơ
sở lý thuyết của về tránh né quảng cáo của các tác giả R. H. Ducoffe [8], L. K. Bracket và B.N. Carr [2], C.H.
Cho và $\mathrm{H}$. J. Cheon [3], D. J. Xu [20], K. Louiseet al. [15], S. M. Choi et al. [4] cũng như từ kết quả phỏng
vấn sơ bộ để đề xuất. Trong đó, tác giả đã phát triển thang đo tránh né trên khía cạnh nhận thức hành vi
dưới sự tác động của giá trị quảng cáo với ba thang đo về giá trị của thông tin, giải trí và khích lệ. Mô hình
được thực hiện với phương pháp phân tích mô hình cấu trúc tuyến tính SEM. Kết quả nghiên cứu cho thây
cả hai thang đo giá trị thông tin và giá trị giải trí đều tác động đến nhận thức và hành vi tránh né. Trong khi
thang đo giá trị thông tin tác động mạnh nhất đến nhận thức thì thang đo giải trí lại tác động mạnh nhất đến
hành vi. Riêng thang đo giá trị khích lệ chỉ tác động đến hành vi tránh né quảng cáo. Từ khóa. giá trị quảng cáo, nhận thức, hành vi, tránh né quảng cáo, mạng xã hội facebook, thành phố Hồ Chí Minh.

\section{THE IMPACT OF ADVERTISING VALUE TO AWARENESS AND BEHAVIOR OF ADVERTISING AVOIDANCE - A STUDY ON FACEBOOK ADVERTISING IN HO CHI MINH CITY}

\begin{abstract}
This research aims to discover and confirm the factors that affect awareness and behaviors that avoid advertising in the Facebook social networking environment. Data were collected from 250 clients living and working in Ho Chi Minh City. The research model and scales were constructed from the theoretical bases of advertising avoidance by R. H. Ducoffe [8], L. K. Bracket và B.N. Carr [2], C.H. Cho và H. J. Cheon [3], D. J. Xu [20], K. Louiseet al. [15], S. M. Choi et al. [4] as well as from the preliminary interview results. Based on that, the author has developed an evasion scale in terms of cognitive behavior under the influence of advertising value with three dimensions of value: information, entertainment and incentive. The model is implemented with SEM linear analytical method. Research results show that both values of information and entertainment value affect avoidance awareness and avoidance behaviors. In the scale of information value has the most impact on perception, the scale of entertainment has the strongest impact on behavior. The incentive value scale only affects the behavior of advertising avoidance.
\end{abstract}

Keywords. advertising value, cognitive, behavior, advertising advoidance, facebook, HoChiMinh city.

\section{1 ĐặT VẤN ĐỀ}

Theo tốc độ phát triển không ngừng của thời đại internet, các trang mạng xã hội trực tuyến ra đời như một sự tất yếu để kết nối người dùng trên phạm vi toàn thế giới. Tại đây, các nhà cung cấp như Facebook, Zalo, Instagram, Myspace, Twitter... không chỉ cung cấp cho người dùng cá nhân một cổng thông tin để thỏa mãn sở thích cá nhân mà còn tạo được một môi trường tương tác hữu hiệu để các doanh nghiệp triển khai hoạt động truyền thông của mình một cách hiệu quả nhất. Trong đó, quảng cáo là một trong những công cự được ứng dụng rộng rãi hơn cả, đặc biệt là thông qua Facebook, nhât là ở thị trường Việt Nam tại thời điểm hiện tại. 
Theo thống kê của Faceboook (2016), Việt Nam có khoảng 30 triệu dân đang sử dụng Facebook với thời gian trải qua trung bình là 2.0 giờ/ ngày. Bên cạnh đó, theo diễn đàn tiếp thị trực tuyến 2016 do Hiệp hội Thương mại điện tử Việt Nam (VECOM) tổ chức, tại Việt Nam, các doanh nghiệp đang chi một nguồn ngân sách làm quảng cáo rất lớn với con số lên đến hàng tỷ đô la. Cụ thể, tại Việt Nam, Facebook hiện đứng số 1 về doanh thu trực tuyến với doanh số khoảng hơn 3.000 tỷ đồng (tương đương 150 triệu USD). Qua số liệu thực tế trên ta thấy các doanh nghiệp Việt Nam rất quan tâm vào hoạt động việc quảng bá cho sản phẩm, dịch vụ của mình trên mạng xã hội Facebook. Tuy nhiên, việc chi nhiều tiền cho quảng cáo như vậy có thật sự mang lại hiệu quả cho các doanh nghiệp không? Bởi vì mỗi cá nhân người dùng có động thái khác nhau đối với hình thức quảng cáo này, có người thì muốn tiếp cận và chấp nhận nhưng có người lại cảm thấy phiền phức nên đã có hành vi tránh né. Việc khảo sát ý kiến của người dùng về quảng cáo nhằm tìm hiểu nguyên nhân tại sao người dùng lại tránh né quảng cáo để có sự thay đổi và đề xuất những chiến lược mới, sáng tạo hơn nhằm thuyết phục người truy cập xem thông tin cần thiết và sự hữu ích do quảng cáo trên Facebook mang đến mà trước giờ họ đã bỏ lỡ.

Liên quan đến vấn đề tránh né quảng cáo, trên thế giới hiện nay có nhiều tác giả bắt đầu quan tâm và đi sâu nghiên cứu về khía cạnh này. Tiên phong là nghiên cứu của tác giả C.H. Cho và H. J. Cheon [3] trong môi trường trực tuyến là với các thang đo xoay quanh góc độ về tiêu cực do quảng cáo mang lại như: cản trở mục tiêu nhận thức, tính hỗn loạn và trải nghiệm tiêu cực từ phía người dùng. Trong đó, tác giả đã khám phá ra rằng tránh né quảng cáo đến từ ba góc độ nhận thức, cảm xúc và hành vi, tuy nhiên tác giả chỉ nghiên cứu chung ba góc độ này trong cùng một thang đo là tránh né. Sau đó, có rất nhiều tác giả ngoài nước đã mở rộng nghiên cứu của mình cho các môi trường cụ thể và bổ sung thêm nhiều thang đo khác. Cụ thể như tác giả K. Louise et al. [15] đã mở rộng nghiên cứu dựa trên nền tảng của C.H. Cho và H. J. Cheon [3] với sự đóng góp của thang đo hướng về thái độ hoài nghi của khách hàng trong môi trường video trực tuyến tác động đến tránh né quảng cáo. Tác giả $\mathrm{D}$. Hussain và $\mathrm{H}$. Lasage [12] lại mở rộng nghiên cứu về tính tương tác tác động đến việc hành vi tránh né quảng cáo trên những trang video trực tuyến. Thông qua các bài báo tham khảo, tác giả nhận thấy rằng, đa phần các bài nghiên cứu chỉ tập trung nghiên cứu cho mạng xã hội nói chung cũng như đa phần nghiên cứu sự tránh né dưới góc độ hành vi $\mathrm{D}$. Hussain và $\mathrm{H}$. Lasage [6] hoặc tránh né nói chung như C.H. Cho và H. J. Cheon [3], K. Louise et al. [15]. Chính vì vậy, tác giả thấy rằng việc nghiên cứu về khía cạnh tránh né quảng cáo trên Facebook tại Việt Nam là thật sự cấp thiết bởi riêng công cụ Facebook, với lượng người dùng khổng lồ và những đặc trưng khác biệt trong quảng cáo sẽ có nhiều yếu tố cần xem xét về tránh né quảng cáo của khách hàng. Đồng thời, tác giả đã mạnh dạn tiên phong đề xuất hướng nghiên cứu về mối quan hệ giữa các giá trị nội tại tích cực mà quảng cáo mang lại tác động đến hai phương diện là nhận thức và hành vi tránh né với đề tài "Tác động của giá trị quảng cáo đến nhận thức và hành vi tránh né quảng cáo - một nghiên cứu về tránh né quảng cáo trên Facebook của khách hàng tại thành phố Hồ Chí Minh".

\section{CƠ SỞ LÝ THUYÊT VÀ MÔ HÌNH NGHIÊN CÚU}

\subsection{Lý thuyết về tránh né quảng cáo}

Tránh né quảng cáo được định nghĩa là "tất cả hành động mà người dùng làm giảm mức độ tiếp xúc của họ với nội dung quảng cáo theo các cách khác nhau" P. S. Speck và T. E. Micheal [19]. Các hành động tránh né này phụ thuộc vào trạng thái tâm lý của khách hàng mà được mở rộng theo ba khía cạnh tiếp cận phổ biến: hành vi, nhận thức và cảm xúc. Việc tránh né dẫn đến ý định bỏ qua quảng cáo của người sử dụng. Tránh né quảng cáo được coi là một trong những trở ngại lớn nhất của quảng cáo.

Theo cách tránh né quảng cáo của P. S. Speck và T. E. Micheal [19] thì tùy thuộc vào đặc điểm cá nhân và các khuôn khổ khác như nhân khẩu học, sự gián đoạn của mục tiêu và các vấn đề liên lạc giữa nhà quảng cáo và người dùng. C.H. Cho và $\mathrm{H}$. J. Cheon [3] tin rằng có ba phương diện khác nhau trong tránh né quảng cáo là tránh né trên phương diện nhận thức, cảm xúc và hành vi.

Khía cạnh hành vi tránh né được xem xét là hành động cuộn qua quảng cáo đó. Việc tránh quảng cáo theo hành vi dẫn đến việc tránh né quảng cáo theo phương thức cơ học, nói cách khác là tránh quảng cáo bằng cách chặn quảng cáo, loại bỏ chúng khỏi bất kỳ trang web nào với ứng dụng trình chặn. Điều này tương đồng với nghiên cứu $\mathrm{W}$. Li và $\mathrm{Z}$. Huang [14] với hành vi quảng cáo trực tuyến với thành phần bao gồm hành vi tránh né thông thường và thông qua sử dụng công cụ hỗ trợ. Đây là một cách hiệu quả để giảm thời gian trôi đi và chặn quảng cáo cho người dùng trực tuyến không có ý định xem nó. Nghiên cứu trước 
đây chứng minh rằng tránh né quảng cáo không bao giờ thành công nếu không có sự hỗ trợ của máy móc. Kỹ thuật tránh né bằng việc sử dụng phần mềm giúp loại bỏ hoàn toàn sự chú ý hay nói cách khác là khách hàng hoàn toàn không biết về sự có mặt của các mẫu quảng cáo.

M. Clancey [5] tuyên bố rằng người tiêu dùng có hành vi tránh né quảng cáo bằng cách "bỏ qua quảng cáo phân tâm", bắt nguồn từ tránh né quảng cáo trên phương diện về nhận thức. Theo C.H. Cho và $\mathrm{H}$. J. Cheon [3] và $K$. Louise et al. [15], thực sự trong số những người dùng tránh né theo phương diện nhận thức cho rằng họ cố ý bỏ qua các quảng cáo, cố ý không nhấn vào các mẫu quảng cáo hay cố tình không chú ý đến các mẫu quảng cáo... Tuy nhiên, các nghiên cứu này cũng cho thấy khách hàng tuy có nhận thức tránh né nhưng thật ra họ vô thức lưu giữ lại một số thông tin quảng cáo.

Tiêu biểu trong khám phá khía cạnh cảm xúc phải kể đển C.H. Cho và H. J. Cheon [3] với các quan sát là cảm thấy ghét quảng cáo và khách hàng sẽ cảm thấy tốt hơn khi không có quảng cáo xuất hiện.

Tuy nhiên, hướng khám phá tránh né dưới góc độ cảm xúc này ít được nhà nghiên cứu tìm hiểu và mở rộng. Riêng đối với hai hướng tránh né về nhận thức và hành vi thì ngược lại. Nó được ứng dụng rộng rãi trong nghiên cứu nhưng thường được các tác giả xét chung trong một thang đo về tránh né quảng cáo như: C.H. Cho và H. J. Cheon [3] và K. Louise et al. [15].

\subsection{Mạng xã hội Facebook}

Theo Wikipedia, mạng xã hội hay gọi là mạng xã hội ảo (tiếng Anh: social network) là dịch vụ nối kết các thành viên cùng sở thích trên Internet lại với nhau với nhiều mục đích khác nhau không phân biệt không gian và thời gian.

Facebook là một website truy cập miễn phí do công ty Facebook, Inc điều hành. Người dùng có thể tham gia các mạng lưới được tổ chức theo thành phố, nơi làm việc, trường học và khu vực để liên kết và giao tiếp với người khác. Mọi người cũng có thể kết bạn và gửi tin nhắn cho họ và cập nhật trang hồ sơ cá nhân của mình để thông báo cho bạn bè biết về chúng. Thành viên đã đăng ký có thể tạo hồ sơ với các hình ảnh, danh sách sở thích cá nhân, thông tin liên lạc, và những thông tin cá nhân khác. Người dùng có thể trao đổi với bạn bè và những người khác thông qua tin nhắn cá nhân hoặc công cộng và tính năng chat, video call, livestream của Facebook. Họ cũng có thể tạo và gia nhập nhóm ưa thích hay "trang yêu thích". Bên cạnh đó, để xoa dịu những lo ngại về sự riêng tư, Facebook cho phép người dùng lựa chọn cài đặt bảo mật của mình và lựa chọn những người có thể nhìn thấy phần cụ thể của tiểu sử của họ. Facebook là một website miễn phí đăng nhập, và nó phát sinh lợi nhuận từ quảng cáo, chẳng hạn thông qua banner quảng cáo. Facebook đòi hỏi tên thành viên và hình ảnh để mọi người có thể đăng nhập vào trang web. Người dùng có thể kiểm soát những ai nhìn thấy các thông tin mà họ đã chia sẻ, cũng như những người có thể tìm thấy chúng trong tìm kiếm, thông qua các thiết lập bảo mật của họ.

\subsection{Mô hình nghiên cứu đề xuất}

Việc tìm hiểu lý do tại sao khách hàng thường tránh né quảng cáo sẽ giúp ích rất lớn cho các doanh nghiệp phát triển các chiến lược quảng bá về hình ảnh công ty cũng như hình ảnh về sản phẩm, dịch vụ mà họ cung cấp. Và chính sự ra đời của các trang mạng xã hội nói chung và mạng Facebook nói riêng đã trở thành một nhà trung gian quảng cáo - phương tiện truyền tải thông điệp mà các doanh nghiệp có thể phát triển. Chính vì lẽ đó mà hiện nay có khá nhiều tác giả nước ngoài trong những năm gần đây đã nghiên cứu về khía cạnh này nhưng tại Việt Nam thì hướng nghiên cứu này còn khá mới và tác giả chưa tìm thấy bài nghiên cứu nào liên quan đến khía cạnh này.

Trong hướng nghiên cứu này, tác giả kế thừa mô hình nghiên cứu của $\mathrm{R}$. H. Ducoffe [7] với giá trị quảng cáo được xem xét dưới hai góc độ là giá trị thông tin và giá trị giải trí. Tuy nhiên khám phá mới của tác giả chính là mở rộng nghiên cứu của R. H. Ducoffe [8] trong môi trường truyền thông trực tuyến sang môi trường mạng xã hội Facebook. Đồng thời, giá trị khích lệ cũng là một góc độ nên được xem xét trong giá trị quảng cáo. Nó được khám phá và kiểm định bởi R. T. Hightower [11] và J. Elisabeth [10]. Với nghiên cứu này, tác giả mạnh dạn tách riêng hai thang đo trong tránh né quảng cáo là nhận thức tránh né và hành vi tránh né trong khám phá mối quan hệ tác động của giá trị quảng cáo. Hai khía cạnh tránh né quảng cáo này đã được rất nhiều tác giả nghiên cứu và áp dụng như C.H. Cho và H. J. Cheon [3], K. Louise et al. [15], W. Li và $Z$. Huang [14] nhưng tất cả các tác giả đều nghiên cứu trong cùng một thang đo là tránh né quảng cáo. Vì những lý do đó, tác giả mạnh dạn đề xuất mô hình nghiên cứu như sau: 


\section{Mô hình nghiên cứu đề xuất của tác giả}

Giá trị quảng cáo

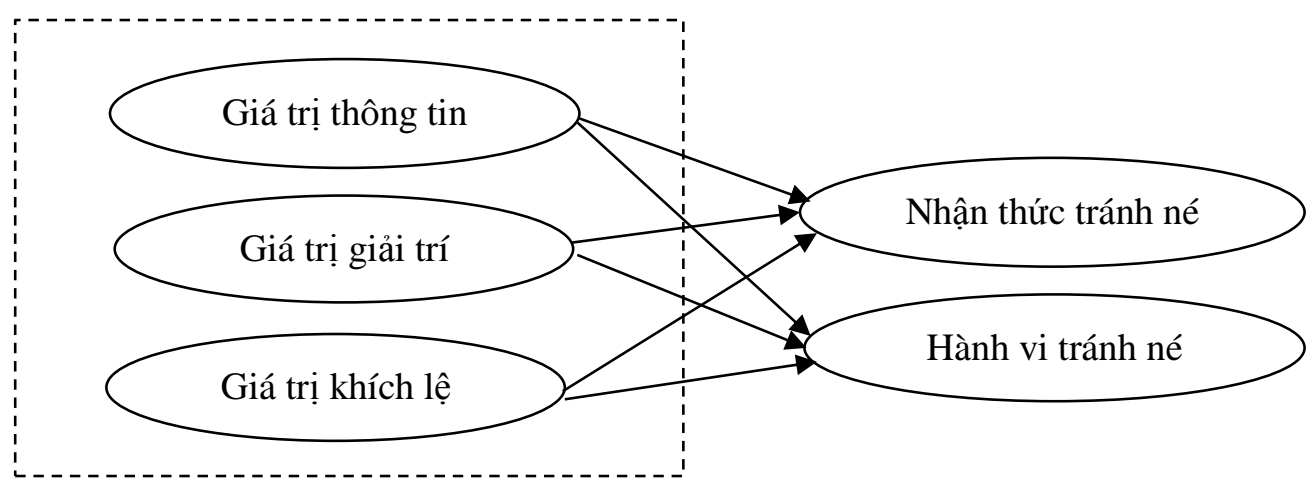

\section{- Giá trị thông tin}

Theo R. H. Ducoffe [7] khi thông điệp của quảng cáo liên quan đến nhu cầu của khách hàng, họ sẽ nhận ra giá trị thông tin mà quảng cáo mang lại. Cũng theo R. H. Ducoffe [8], giá trị thông tín của quảng cáo trực tuyến được đo lường bằng: sự hữu ích, quan trọng, cung cấp nhiều tin tức. Thang đo này sau đó đã được S. M. Edwards et al. [9] kiểm định thực nghiệm trong nghiên cứu của mình. Tính cập nhật của thông tin được L. K. Bracket và B.N. Carr [2] chỉ ra trong nghiên cứu của mình khi thực nghiệm trong trường hợp quảng cáo thông qua thiết bị di động.

Trên thực tế, công cụ quảng cáo mà Facebook hiện đang cung cấp cho các doanh nghiệp luôn không ngừng được tối ưu hóa. Facebook sẽ ưu tiên hiển thị quảng cáo của doanh nghiệp cho những người sẽ chú ý đến nó thông qua chức năng khảo sát đặc điểm cá nhân và lịch sử view, like, comment, share.. của đối tượng người dùng đối với các trang quảng cáo tương tự để phân phối các quảng cáo. Do đó, tác giả đề xuất thang đó giá trị thông tin đúng lúc vào mô hình thang đo để đánh giá hiệu quả của công cụ này. Khi đó, những thông tin tương tự hoặc có nội dung gần gũi mà quảng cáo trên Facebook đề xuất sẽ phù hợp với những gì mà người dùng tìm kiếm.

S. Lee và $\mathrm{J}$. R. Lumpkin [13] khi nghiên cứu về quảng cáo truyền hình đã chứng minh được hành vi tránh né quảng cáo của người xem được điều chỉnh bởi giá trị của thông tin mà quảng cáo đó chứa đựng. D. S. Aaker và E. B. Donald [1], sau đó là Y. Pasadeos [17] với nghiên cứu của mình đã chỉ ra rằng quảng cáo được nhận thức có giá trị thông tin ít gây ra hành vi tránh né hơn những quảng cáo không có giá trị thông tin hữu ích. Phát triển nghiên cứu của Choi và Cheon (2004) trong môi trường mạng xã hội, K. Louise et al. [15] nghiên cứu trên đối tượng là giới trẻ, đã chứng minh được nếu thông tin không mang lại những giá trị cho người xem thì họ sẽ tránh né quảng cáo, trong đó, tránh né được tác giả đo lường qua hai khía cạnh: hành vi và nhận thức. Theo đó, giả thuyết được đặt ra như sau:

H1: Giá trị thổng tin có tác động nghịch chiểu đến nhận thức tránh né quảng cáo của người dùng Facebook

H2: Giá trị thông tin có tác động nghịch chiều đến hành vi tránh né quảng cáo của người dùng Facebook

\section{- Giá trị giải trí}

R. H. Ducoffe [7] đã thực hiện nghiên cứu và chứng minh được sự ảnh hưởng của giá trị giải trí khán giả xem quảng cáo trên các phương tiện truyền thông truyền thống. Sau đó, R. H. Ducoffe [8] mở rộng nghiên cứu của mình sang lĩnh vưc trực tuyến và cũng chứng minh được kết quả tương tự. Thang đo giá trị giải trí được tác giả kế thừa của $\mathrm{A}$. M. Rubin et al. [18] bao gồm ba yếu tố: trải nghiệm vui vẻ, cảm giác giải trí và bị thu hút đối với các mẫu quảng cáo. Dựa theo nghiên cứu này A.N. Diaz et al. [6], S. M. Edwards et al. [9], C.H. Cho và H. J. Cheon [3] đã kiểm định được mối quan hệ nghịch chiều giữa giá trị giải trí và hành vi tránh né quảng cáo của người dùng internet. Còn theo $\mathrm{D}$. J. Xu [20] thì giá trị giải trí liên quan đến sự thích thú với thông điệp mà quảng cáo mang lại. C.H. Cho và $\mathrm{H}$. J. Cheon [3] nghiên cứu trường hợp video quảng cáo trực tuyến đã chỉ ra rằng nếu người dùng cảm nhận tính giải trí càng cao thì hành vi tránh né quảng cáo càng khó xảy ra. Tác giả S. M. Choi et al. [4] cũng đã kiểm định được giá trị 
giải trí là yếu tố tác động âm và có mức độ tác động mạnh nhất đến nhận thức tránh né quảng cáo của người dùng internet và được đo lường bởi việc người dùng cảm thấy bị thu hút, được giải trí và vui vẻ khi xem quảng cáo và tìm ra thêm một biến quan sát nữa là được tận hưởng khi xem quảng cáo. Theo đó, giả thuyết $\mathrm{H}_{3}, \mathrm{H}_{4}$ được đặt ra cho nghiên cứu như sau:

$\mathrm{H}_{3}$ : Giá trị giải trí có tác động nghịch chiều đến nhận thức tránh né quảng cáo của người dùng Facebook

$\mathrm{H}_{4}$ : Giá trị giải trí có tác động nghịch chiều đến hành vi tránh né quảng cáo của người dùng Facebook

\section{- Giá trị khích lệ}

Jones (2009) đã chứng minh được rằng người dùng ý thức được việc mình có thể xem video chất lượng cao từ các website chính là nhờ sự tài trợ kinh phí từ các mẫu quảng cáo. C.H. Cho và H. J. Cheon [3] khi nghiên cứu về video quảng cáo trực tuyến đã cho thấy nếu người dùng cảm nhận mình phải nhận càng ít các quảng cáo khi xem các video chất lượng tốt thì khả năng tránh né quảng cáo càng thấp.

Những kết quả nêu trên được những nhà nghiên cứu thực nghiệm trong môi trường video quảng cáo trực tuyến. Kế thừa vào đề tài này, với quảng cáo qua công cụ Facebook, tác giả nhận thấy có sự phù hợp về phương diện quảng cáo trực tuyến. Tuy nhiên, để làm rõ đặc trưng của mạng xã hội với tính năng liên kết và chia sẻ giữa những người dùng, tác giả đề xuất thêm thang đo nhận biết được nhiều xu hướng/ trào lưu...mà bạn bè và những người xung quanh quan tâm khi xem các mẫu quảng cáo trên Facebook. Đồng thời, tác giả cũng mạnh dạn đề xuất thêm hai thang đo: người dùng nhận thấy mình biết được rất nhiều các chương trình ưu đãi/ giảm giá và trở thành người tiêu dùng thông minh nhờ xem các mẫu quảng cáo trên Facebook để kiểm định về tác động của các chương trình truyền thông tích hợp mà các doanh nghiệp kinh doanh nên triển khai để nâng cao hiệu quả của hoạt đông quảng cáo thông qua trang mạng xã hội này.

Kết quả nghiên cứu R. T. Hightower [11] về hành vi tránh né quảng cáo trực tuyến cho thấy nếu quảng cáo càng có giá trị khích lệ thì khả năng tránh né của người xem càng giảm thiểu. Về giá trị khích lệ, nghiên cứu của C.H. Cho và $\mathrm{H}$. J. Cheon [3] cũng đã đo lường giá trị này trong thang đo về trải nghiệm tiêu cực và chỉ ra tác động ngược chiều của giá trị khích lệ đến tránh né quảng cáo thông qua ba khía cạnh: nhận thức, hành vi, tình cảm. Do đó, giả thuyết được đặt ra như sau:

$\mathrm{H}_{5}$ : Giá trị khích lệ có tác động nghịch chiều đến nhận thức tránh né quảng cáo của người dùng Facebook

H63: Giá trị khích lệ có tác động nghịch chiều đến hành vi tránh né quảng cáo của người dùng Facebook

\section{PHƯƠNG PHÁP NGHIÊN CÚU}

\subsection{Qui trình nghiên cứu}

Nghiên cứu được thực hình thông qua hai bước là nghiên cứu sơ bộ và nghiên cứu chính thức thông qua hai phương pháp:

Nghiên cứu sơ bộ bằng phương pháp nghiên cứu định tính với mục đích tìm hiểu xem người được phỏng vấn có hiểu được nội dung, cũng như bổ sung, hiệu chỉnh hay loại bỏ bớt các phát biểu để hình thành thang đó chính thức. Phương pháp này được thực hiện bằng kỹ thuật phỏng vấn tay đôi với 10 khách hàng cả hai giới tính nam và nữ với các độ tuổi khác nhau đang học tập và làm việc tại thành phố Hồ Chí Minh. Các phát biểu cuối cùng được tổng hợp lại thành thang đo chính thức làm cơ sở cho nghiên cứu định lượng.

Nghiên cứu chính thức bằng phương pháp nghiên cứu định lượng được thực hiện đầu tiên với phân tích độ tin cậy bằng hệ số Cronbach's Alpha cho từng thang đo đơn hướng. Kiểm tra này nhằm đảm bảo các thang đo đơn hướng đều có hệ số Cronbach's Alpha lớn hơn 0.6 nhưng không lớn hơn 0.95 . Những biến có hệ số tương quan biến tổng nhỏ hơn 0.3 sẽ bị loại khỏi thang đo $(T$. Đ. Nguyễn và $T$. T. M. Nguyễn [16]). Phương pháp phân tích nhân tố khám phá EFA với phương pháp trích Principal Axis Factoring với phép quay Promax nhằm phản ánh cấu trúc dữ liệu chính xác hơn được sử dụng với điều kiện đạt tin cậy là hệ số tải nhân tố Factor loading $\geq 0.5$, hệ số tương quan $\geq 0.3$, tổng phương sai trích $\geq 50 \%$ và $\mathrm{KMO} \geq 0.5$, kiểm định Bartlett có ý nghĩa thống kê, Sig < 0.05 (T. Đ. Nguyễn và T. T. M. Nguyễn [16]). Phương pháp phân tích nhân tố khẳng định CFA trong phân tích mô hình cấu trúc tuyến tính SEM: nhằm kiểm định xem mô hình có đạt yêu cầu hay không và thang đo có đạt yêu cầu của thang đo tốt hay không. Để đo lường mức độ phù hợp của mô hình với thông tin thị trường, các chỉ tiêu quan tâm là (Nguyễn \& Nguyễn 2011): (1) phương pháp ước lượng là Maximum Likelihood, (2) Chi-bình phương có giá trị p > 5\%, (3) chỉ số TLI, và $C F I$ đều $\geq 0.9$. (4) Chi-bình phương điều chỉnh theo bậc tự do $C M I N / d f \leq 3$ và (5) chỉ số $R M S \leq 0.08$. 
(T. Đ. Nguyễn và T. T. M. Nguyễn [16]). Các chỉ tiêu đánh giá thang đo là hệ số tin cậy tổng hợp, tổng phương sai trích được, tính đơn hướng, giá trị hội tụ và giá trị phân biệt. Cuối cùng, phương pháp Bootstrap được sử dụng để ước lượng lại các tham số mô hình và kiểm định độ tin cậy của các ước lượng.

\subsection{Thang đo}

Tất cả các thang đo các khái niệm nghiên cứu trong mô hình đều là thang đo đơn hướng. Các thang đo này sử dụng dạng Likert, năm điểm với 1 : hoàn toàn không đồng ý và 5 : hoàn toàn đồng ý. Thang đo tránh né quảng cáo bao gồm hai thành phần là hành vi tránh né $(\mathrm{HV})$ và nhận thức tránh né $(\mathrm{NT})$ với nhân tố tác động là giá trị quảng cáo với cụ thể ba thành phần là giá trị thông tin (TT), giá trị giải trí $(\mathrm{GT})$ và giá trị khích lệ (KL).

Trong đó, nội dung của từng thành phần được tham khảo từ các tác giả R. H. Ducoffe [8], L. K. Bracket và B.N. Carr [2], C.H. Cho và H. J. Cheon [3], D. J. Xu [20], S. M. Choi et al. [4] và từ kết quả phỏng vấn sơ bộ để đề xuât.

Bảng 1. Bảng thống kê nguồn của biến quan sát

\begin{tabular}{|c|c|c|}
\hline $\begin{array}{l}\text { Mã } \\
\text { hóa }\end{array}$ & Thang đo & Nguồn \\
\hline \multicolumn{3}{|c|}{ GIÁ TRỊ THÔNG TIN_TT } \\
\hline TT1 & Tôi thấy các mẫu quảng cáo trên Facebook mang đến những thông tin hữu ích & R. H. Ducoffe [8] \\
\hline TT2 & $\begin{array}{l}\text { Tôi thấy các mẫu quảng cáo trên Facebook mang đến những thông tin quan } \\
\text { trọng }\end{array}$ & R. H. Ducoffe [8] \\
\hline TT3 & Tôi thấy các mẫu quảng cáo trên Facebook mang đến nhiều thông tin & R. H. Ducoffe [8] \\
\hline TT4 & Các mẫu quảng cáo trên Facebook giúp tôi cập nhật thông tin liên tục & $\begin{array}{l}\text { L. K. Bracket và B.N. } \\
\text { Carr [2] }\end{array}$ \\
\hline TT5 & $\begin{array}{l}\text { Tôi thấy các mẫu quảng cáo trên Facebook mang đến những thông tin phù hợp } \\
\text { với nhu cầu cưa tôi }\end{array}$ & Tác giả đề xuất \\
\hline \multicolumn{3}{|c|}{ GIÁ TRI GIẢI TRÍ_GT } \\
\hline GT1 & Tôi cảm thấy bị thu hút bởi các mẫu quảng cáo trên Facebook & R. H. Ducoffe [8] \\
\hline GT2 & Tôi thấy các mẫu quảng cáo trên Facebook mang tính giải trí cao & R. H. Ducoffe [8] \\
\hline GT3 & Tôi cảm thấy vui vẻ khi xem các mẫu quảng cáo trên Facebook & R. H. Ducoffe [8] \\
\hline GT4 & Tôi cảm thấy vui vẻ khi xem các mẫu quảng cáo trên Facebook & D. J. Xu [20] \\
\hline GT5 & Tôi tận hưởng thời gian xem các mẫu quảng cáo trên Facebook & S. M. Choi et al. [4] \\
\hline \multicolumn{3}{|c|}{ GIÁ TRỊ KHÍCH LẸ_KL } \\
\hline KL1 & $\begin{array}{l}\text { Tôi nhận thấy mình được các công ty đăng quảng cáo trên Facebook hỗ trợ chi } \\
\text { phí cho việc sử dụng miễn phí ứng dụng này }\end{array}$ & $\begin{array}{lrrr}\text { S. } & \text { Lee và } & \text { J. } & \text { R. } \\
\text { Lumpkin [13] } & \end{array}$ \\
\hline KL2 & $\begin{array}{l}\text { Tôi nhận thấy mình được xem các thông tin/ video/... có chất lượng với rất ít } \\
\text { các mấu quảng cáo trên Facebook }\end{array}$ & $\begin{array}{l}\text { C.H. Cho và H. J. } \\
\text { Cheon [3] }\end{array}$ \\
\hline KL3 & $\begin{array}{l}\text { Tôi nhâan thấy mình biết được rất nhiều các chương trình ưu đãi/ giảm giá... } \\
\text { khi xem các mẫu quảng cáo trên Facebook }\end{array}$ & Tác giả đề xuất \\
\hline KL4 & $\begin{array}{l}\text { Tôi biết được nhiều xu hướng/ trào lưu... mà bạn bè và những người xung } \\
\text { quanh quan tâm khi xem các mấu quảng cáo trên Facebook }\end{array}$ & Tác giả đề xuất \\
\hline KL5 & $\begin{array}{l}\text { Tôi nhận thấy mình trở thành người tiêu dùng thông minh nhờ xem các mẫu } \\
\text { quảng cáo trên Facebook }\end{array}$ & Tác giả đề xuất \\
\hline \multicolumn{3}{|c|}{ NHậN THỨC TRÁNH NÉ_NT } \\
\hline NT1 & Tôi cố ý bỏ qua những mẫu quảng cáo trên Facebook & K. Louise et al. [15] \\
\hline NT2 & Tôi không bao giờ nhấn vào bất kỳ mẫu quảng cáo nào trên Facebook & K. Louise et al. [15] \\
\hline NT3 & Tôi không chú ý vào những mẫu quảng cáo trên Facebook & K. Louise et al. [15] \\
\hline \multicolumn{3}{|c|}{ HÀNH VI TRÁNH NÉ_HV } \\
\hline HV1 & Tôi cuộn xuống khỏi những mẫu quảng cáo trên Facebook & K. Louise et al. [15] \\
\hline HV2 & Tôi gửi phản hồi với Facebook để từ chối những mẫu quảng cáo & Tác giả đề xuất \\
\hline HV3 & Tôi cài đặt phần mềm để hạn chế các quảng cáo trên Facebook & Tác giả đề xuất \\
\hline HV4 & Tôi dùng các thủ thuật để hạn chế những mẫu quảng cáo trên Facebook & Tác giả đề xuất \\
\hline
\end{tabular}

Nguồn: Tác giả tự tổng hơp 


\subsection{Mô tả mẫu nghiên cứu}

Nghiên cứu này sử dụng kỹ thuật phỏng vấn bằng bảng câu hỏi được thiết kế trên form Google Docs với khách hàng cả hai nhóm giới tính nam và nữ tại các độ tuổi khác nhau trên địa bàn TPHCM. Mẫu được chọn theo phương pháp ngẫu nhiên đơn giản và không theo tỉ lệ với kích thước $\mathrm{n}=250$. Sau khi thu thập, ta có thành phần mẫu như sau: nam giới chiếm $46.8 \%$ và nữ giới chiếm $53.2 \%$ với tỷ lệ độ tuổi là $30.4 \%$ khách hàng có độ tuổi dưới 25 tuổi, $43.6 \%$ khách hàng ở độ tuổi 25 - 40 tuổi, và trên 40 tuổi chiếm $26 \%$.

\section{PHÂN TÍCH DŨ̃ LIỆU VÀ KẾT QUẢ}

\subsection{Phân tích độ tin cậy của thang đo}

Bảng 2. Kết quả phân tích độ tin cậy Cronbach’s Alpha

\begin{tabular}{|c|c|c|c|c|}
\hline Biến quan sát & $\begin{array}{l}\text { Trung bình thang đo nếu } \\
\text { loại biến }\end{array}$ & $\begin{array}{l}\text { Phương sai thang đo } \\
\text { nếu loại biến }\end{array}$ & Tương quan biến tổng & $\begin{array}{l}\text { Crobach Alpha nếu } \\
\text { loại biến }\end{array}$ \\
\hline \multicolumn{5}{|c|}{ Giá trị thông tin: $\alpha=0.886$} \\
\hline TT1 & 15.27 & 5.947 & 642 & .882 \\
\hline TT2 & 15.32 & 5.415 & .800 & .843 \\
\hline TT3 & 15.36 & 5.733 & .763 & .853 \\
\hline TT4 & 15.38 & 5.916 & .782 & .850 \\
\hline TT5 & 15.38 & 6.327 & .653 & .877 \\
\hline \multicolumn{5}{|c|}{ Giá trị giải trí: $\alpha=0.875$} \\
\hline GT1 & 14.14 & 6.658 & .595 & .873 \\
\hline GT2 & 14.16 & 5.939 & .733 & .841 \\
\hline GT3 & 14.09 & 6.099 & .771 & .833 \\
\hline GT4 & 14.19 & 5.724 & .757 & .835 \\
\hline GT5 & 14.08 & 6.199 & .671 & .857 \\
\hline \multicolumn{5}{|c|}{ Giá trị khích lệ: $\alpha=0.881$} \\
\hline KL1 & 14.93 & 5.924 & .727 & .852 \\
\hline KL2 & 14.99 & 5.598 & .749 & .847 \\
\hline KL3 & 15.03 & 5.758 & .742 & .848 \\
\hline KL4 & 15.08 & 6.003 & .686 & .862 \\
\hline KL5 & 15.12 & 6.110 & .667 & .866 \\
\hline \multicolumn{5}{|c|}{ Nhận thức tránh né: $\boldsymbol{\alpha}=0.838$} \\
\hline NT1 & 4.42 & 2.049 & 670 & .804 \\
\hline NT2 & 4.45 & 1.982 & .753 & .726 \\
\hline NT3 & 4.44 & 1.910 & .682 & .795 \\
\hline \multicolumn{5}{|c|}{ Hành vi tránh né: $\alpha=0.867$} \\
\hline HV1 & 7.38 & 4.381 & 619 & .874 \\
\hline HV2 & 7.45 & 4.446 & .739 & .824 \\
\hline HV3 & 7.42 & 4.192 & .826 & .789 \\
\hline HV4 & 7.57 & 4.194 & .710 & .835 \\
\hline
\end{tabular}

Nguồn: Kết quả khảo sát của tác giả

Kết quả phân tích cho thấy tất cả các thang đo đều có hệ số Crobach's Alpha $>0.06$ (cụ thể hệ số Crobach's Alpha dao động từ 0.838 - 0.886), đồng thời hệ số tương quan biến - tổng khi loại biến của các biến quan sát cho từng thang đo đều lớn hơn 0.03 . Vì vậy tất cả các biến quan sát và thang đo đều đạt yêu cầu để đưa vào tiếp tục phân tích nhân tố khám phá EFA.

\subsection{Phân tích nhân tố khám phá EFA}

Bài nghiên cứu này sử dụng phương pháp trích trong phân tích nhân tố chính (Principal Axis Analysis) cùng phép xoay áp dụng là Promax. Kết quả EFA cho thấy giá trị KMO đạt được là $0.859>0.05$ và giá trị $\mathrm{Sig}=0.000$ (chứng tỏ các biến quan sát có tương quan với nhau trong tổng thể). Đồng thời kết quả cho thây có 5 nhân tố tại Eigen value lớn hơn 1 và tổng phương sai trích tích lũy 63.397 (> 50\%, cho biết 5 nhân tố rút trích ra giải thích được $63.397 \%$ biến thiên của dữ liệu). 
Kết quả phân tích nhân tố khám phá EFA ta thấy có 5 nhân tố trích được với các biến quan sát không bị xáo trộn trong từng thang nên ta giữ lại tên thang đo như cũ, cụ thể là: giá trị thông tin (TT1-TT5), giá trị giải trí (GT1-GT5), giá trị khích lệ (KL1-KL5), nhận thức tránh né (NT1-NT3), hành vi tránh né (HV1HV4).

Sau khi phân tích nhân tố EFA, phân tích nhân tố khẳng định CFA được sử dụng kể kiểm định thang đo các khái niệm nghiên cứu và mô hình cấu trúc tuyến tính SEM được dùng để kiểm định mô hình lý thuyết và các giả thuyết.

\subsection{Mô hình đo lường tới hạn CFA}

Vì các khái niệm sử dụng trong mô hình lý thuyết là các khái niệm đơn hướng, vì vậy chúng được đánh giá trong cùng một mô hình đo lường tới hạn CFA.

Theo kết quả thống kê cho thấy mô hình tới hạn có 199 bậc tự do. Kết quả CFA cho thấy Chi-bình phương $=244.814, \mathrm{p}=.015$, Chi-bình phương $/ \mathrm{df}=1.203(<3), \mathrm{TLI}=0.962, \mathrm{CFI}=.967$ (đều >0.9), RMSE $=.045(<0.08)$ nên mô hình phù hớp với dữ liệu thị trường. Đồng thời kết quả cũng cho thấy trọng số $\mathrm{CFA}$ của tất cả các biến quan sát đều lớn hơn 0.05 (nhỏ nhất là 0.59 ) khẳng định tính đơn hướng và giá trị hội tụ của các thang đo được sử dụng trong mô hình nghiên cứu.

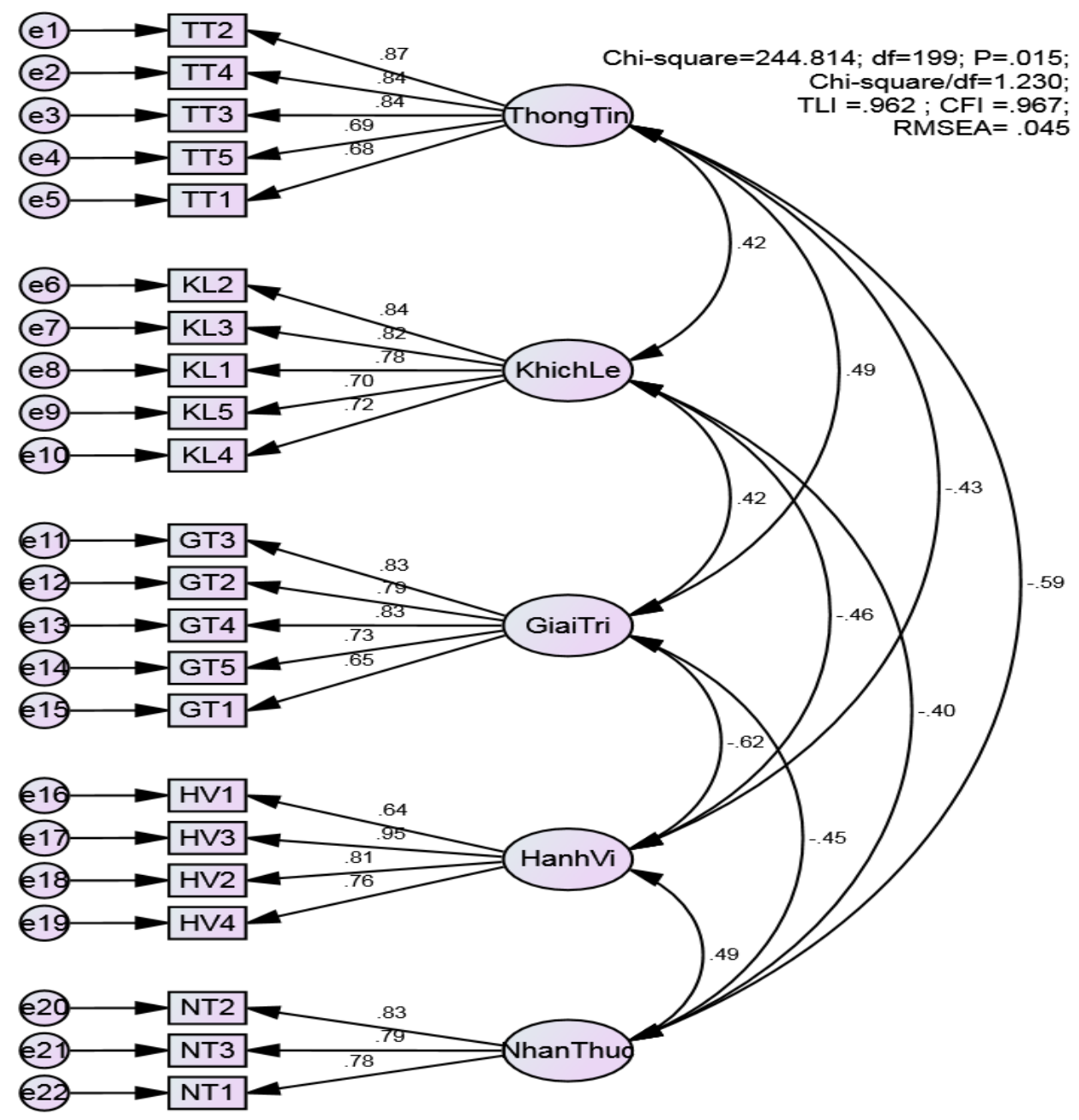

Hình 1. Kết quả mô hình đo lường tới hạn CFA

Kết quả cho ta thấy tất cả các hệ số tương quan giữa các khái niệm đều nhỏ hơn 1 với các giá trị p < 0.05. Tóm lại, tất cả các thang đo đều đạt được giá trị phân biệt với mức ý nghĩa thống kê $95 \%$. 
102 TÁC ĐộNG CỦA GIÁ TRİ QUẢNG CÁO ĐẾN NHẬN THỨC VÀ HÀNH VI TRÁNH NÉ QUẢNG CÁO MỘT NGHIÊN CUUUU VỀ TRÁNH NÉ QUẢNG CÁO TRÊN FACEBOOK TẠI TP. HỒ CHÍ MINH

Bảng 3: Bảng thể hiện giá trị mối quan hệ của các thang đo

\begin{tabular}{|c|c|c|c|c|}
\hline Mối quan hệ & $\mathrm{r}$ & se & $\mathrm{cr}$ & $\mathrm{p}$ \\
\hline ThongTin <--> GiaiTri & .579 & .045 & 4.194 & $* * *$ \\
\hline ThongTin $<-->$ KhichLe & .426 & .044 & 3.355 & $* * *$ \\
\hline ThongTin <--> HanhVi & -.569 & .048 & -4.052 & $* * *$ \\
\hline ThongTin <--> NhanThuc & -.614 & .046 & -4.303 & $* * *$ \\
\hline GiaiTri <--> KhichLe & .473 & .048 & 3.571 & $* * *$ \\
\hline GiaiTri <--> HanhVi & -.617 & .053 & -4.189 & $* * *$ \\
\hline GiaiTri <--> NhanThuc & -.464 & .046 & -3.457 & $* * *$ \\
\hline KhichLe <--> HanhVi & -.388 & .049 & -3.048 & .002 \\
\hline KhichLe <--> NhanThuc & -.318 & .045 & -2.530 & .011 \\
\hline HanhVi <--> NhanThuc & .607 & .053 & 4.101 & $* * *$ \\
\hline
\end{tabular}

Nguồn: Kết quả khảo sát của tác giả

\subsection{Kiểm định độ tin cậy tổng họ̣p và phương sai trích}

Độ tin cậy tổng hợp $\mathrm{p}_{\mathrm{c}}$ và phương sai trích $\mathrm{p}_{\mathrm{vc}}$ được tính trên cơ sở trọng số nhân tố ước lượng trong các mô hình CFA của các thang đo. Kết quả cho thấy độ tin cậy tổng hợp và phương sai trích của các thang đo đều $>0.50$ nên ta có thể kết luận các thang đo đều đạt yêu cầu về giá trị độ tin cậy.

Bảng 4. Bảng tóm tắt kết quả kiểm định độ tin cậy tổng hợp và phương sai trích

\begin{tabular}{|c|c|c|c|c|c|c|}
\hline Khái niệm & $\begin{array}{l}\text { Số } \\
\text { thành } \\
\text { phần }\end{array}$ & $\begin{array}{l}\text { Số biến } \\
\text { quan sát }\end{array}$ & $\begin{array}{l}\text { Độ tin cậy } \\
\text { Cronbach's } \\
\text { Alpha }\end{array}$ & $\begin{array}{l}\text { Độ tin cậy } \\
\text { tổng hợp }\end{array}$ & $\begin{array}{l}\text { Phương } \\
\text { sai trích }\end{array}$ & Giá trị \\
\hline ThongTin & 1 & 5 & 0.886 & 0.780 & $59.9 \%$ & \multirow{5}{*}{$\begin{array}{l}\text { Chấp } \\
\text { nhận }\end{array}$} \\
\hline GiaiTri & 1 & 5 & 0.875 & 0.852 & $53.9 \%$ & \\
\hline KhichLe & 1 & 5 & 0.881 & 0.863 & $56.0 \%$ & \\
\hline HanhVi & 1 & 4 & 0.867 & 0.894 & $68.0 \%$ & \\
\hline NhanThuc & 1 & 3 & 0.838 & 0.831 & $62.1 \%$ & \\
\hline
\end{tabular}

Nguồn: Kết quả khảo sát của tác giả

\subsection{Kiểm định mô hình lý thuyết và giả thuyết nghiên cứu bằng SEM}

\section{- Kiểm định mô hình lý thuyết}

Kết quả phân tích SEM cho thấy mô hình có Chi-bình phương $=296.257$ với 203 bậc tự do và $\mathrm{p}=$ .000. Các hệ số khác như Chi-bình phương/df $=1.459(<3), \mathrm{TLI}=.924, \mathrm{CFI}=.933$ (đều >0.9) và RMSEA $=.064(<.08)$ đều đạt chỉ tiêu. Như vậy ta hoàn toàn có thể kết luận là mô hình này phù hợp với các dữ liệu thị trường thu thập được. 


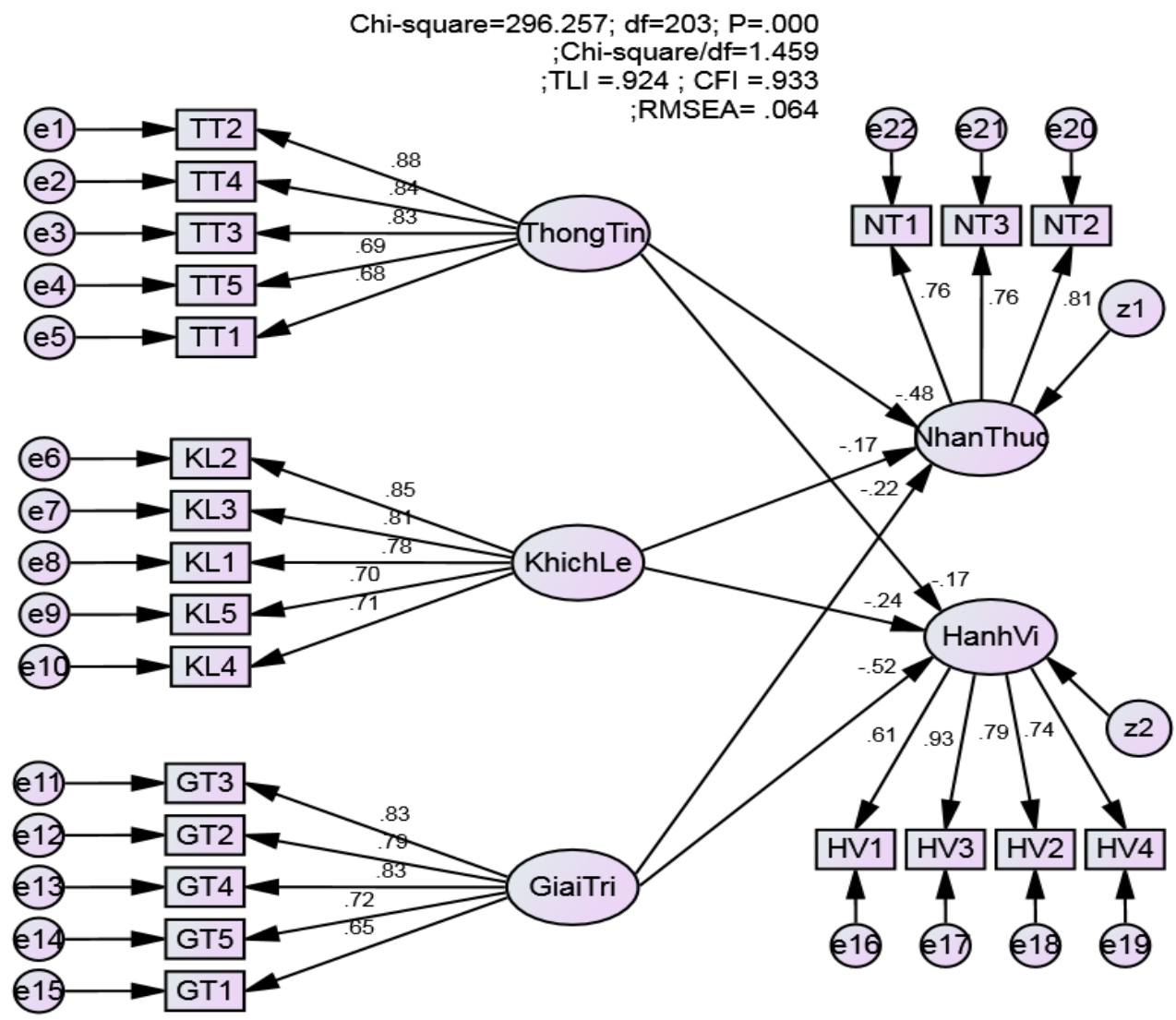

Hình 2. Kết quả SEM của mô hình lý thuyết (chuẩn hóa)

\section{- Kiểm định ước lượng mô hình lý thuyết bằng bootstrap}

Các ước lượng cho mô hình lý thuyết được kiểm định bằng phương pháp Bootstrap với số lượng mẫu lặp lại $\mathrm{N}=1000$. Kết quả ước lượng cho thấy có xuất hiện độ chệch, tuy nhiên trị tuyệt đối tất cả của các độ chệch đều nhỏ hơn hoặc bằng 3.33. Như vậy, ta có thể đi đến kết luận là những ước lượng trong mô hình có thể tin cậy được

Bảng 5. Kết quả của ước lượng bằng bootstrap với $\mathrm{N}=1000$

\begin{tabular}{|l|r|r|r|r|r|r|r|}
\hline Mối quan hệ & $\begin{array}{c}\text { Uớc lượng } \\
\text { ML }\end{array}$ & SE & SE-SE & Mean & Bias & SE-Bias & CR \\
\hline NhanThuc <--- ThongTin & -.482 & .118 & .003 & -.472 & .009 & .004 & 2.25 \\
\hline NhanThuc <--- KhichLe & -.171 & .129 & .003 & -.176 & -.005 & .004 & -1.25 \\
\hline NhanThuc <--- GiaiTri & -.220 & .116 & .003 & -.219 & .001 & .004 & 0.25 \\
\hline HanhVi <--- ThongTin & -.165 & .116 & .003 & -.168 & -.002 & .004 & -0.50 \\
\hline HanhVi <--- KhichLe & -.244 & .134 & .003 & -.245 & -.001 & .004 & -0.25 \\
\hline HanhVi <--- GiaiTri & -.518 & .112 & .003 & -.500 & .008 & .004 & 2.00 \\
\hline
\end{tabular}

Nguồn: Kết quả khảo sát của tác giả 


\section{- Kiểm định giả thuyết}

Bảng 6. Kiểm định mối quan hệ giữa các khái niệm trong mô hình nghiên cứu (chuẩn hóa)

\begin{tabular}{|c|l|r|r|r|r|}
\hline Giả thuyết & \multicolumn{1}{|c|}{ Mối quan hệ } & Estimate & S.E. & C.R. & P \\
\hline H1 & NhanThuc <--- ThongTin & -.407 & .088 & -4.626 & $* * *$ \\
\hline H5 & NhanThuc <--- KhichLe & -.146 & .084 & -1.745 & .081 \\
\hline H3 & NhanThuc <--- GiaiTri & -.215 & .097 & -2.226 & .026 \\
\hline H2 & HanhVi <--- ThongTin & -.123 & .068 & -1.822 & .039 \\
\hline H6 & HanhVi <--- KhichLe & -.185 & .072 & -2.571 & .010 \\
\hline H4 & HanhVi <--- GiaiTri & -.449 & .100 & -2.510 & $* * *$ \\
\hline
\end{tabular}

Nguồn: Kết quả khảo sát của tác giả

Kết quả ước lượng chưa chuẩn hóa của các tham số chính trong mô hình lý thuyết và kết quả chuẩn hóa cho ta thấy kết quả mối quan hệ giữa giá trị khích lệ và nhận thức tránh né không đạt mức ý nghĩa thống kê ( $p>0.05)$. Vì vậy giả thuyết H5 không được chấp nhận. Các mối quan hệ còn lại đều có ý nghĩa thống kê (với $\mathrm{p}<0.05$ ), nghĩa là các giải thuyết $\mathrm{H} 1, \mathrm{H} 2, \mathrm{H} 3, \mathrm{H} 4$ và $\mathrm{H} 6$ đều được chấp nhận. Cụ thể giá trị thông tin có tác động mạnh nhất đến nhận thức tránh né (với $\beta=-0.482$ ), nhưng lại tác động thấp nhất đối với hành vi tránh né (với $\beta=-0.165$ ). Còn đối với giá trị giải trí thì hoàn toàn ngược lại, nó tác động mạnh nhất đến hành vi tránh né quảng cáo (với $\beta=-0.518$ ) và yếu nhất tới nhận thức (với $\beta=-0.220$ ). Riêng giá trị khích lệ chỉ tác động đến hành vi tránh né (với mức $\beta=-0.244$ ).

\section{HÀM Ý CỦA NGHIÊN CỨU VÀ KIẾN NGHỊ CHO NHÀ QUẢN TR!}

Nghiên cứu này cho thấy, thang đo về tránh né quảng cáo của các tác giả nước ngoài thực nghiệm cho các trường hợp video quảng cáo trực tuyến có thể áp dụng cho trường hợp chuyên biệt của công cụ Facebook. Tuy nhiên trong trường hợp này, chúng ta cần một số điều chỉnh và bổ sung cho phù hợp. Kết quả nghiên cứu chỉ ra rằng trong ba giá trị thuộc giá trị quảng cáo được đưa ra và kiểm định đều tác động đến hành vi tránh né quảng cáo, trong đó giá trị giải trí là có ảnh hưởng mạnh nhất. Tuy khác nhau trong môi trường nghiên cứu, kết quả này đạt được sự tương đồng với tác giả $\mathrm{S}$. Lee và $\mathrm{J}$. R. Lumpkin [13], D. $S$. Aaker và $E$. $B$. Donald [1], sau đó là $Y$. Pasadeos [17] rằng hành vi tránh né quảng cáo và giá trị thông tin có mối quan hệ nghịch biến hay giá trị giải trí là yếu tố tác động âm và có mức độ tác động mạnh nhất đến hành vi tránh né quảng cáo (S. M. Choi et al. [4]) hay mối quan hệ nghịch biến giữa hành vi và giá trị khích lệ R. T. Hightower [11].

Đóng góp đáng kể của tác giả chính là nghiên cứu mối quan hệ của giá trị quảng cáo đến nhận thức tránh né mà các tác giả khác còn bỏ ngỏ. Kết quả cho thấy chỉ có giá trị thông tin và giá trị giải trí có ảnh hưởng đến nhận thức tránh né quảng cáo còn giá trị khích lệ thì không. Bên cạnh đó, nghiên cứu cũng khám phá thêm rằng các giá trị thang đo như: sự phù hợp giữa thông tin và nhu cầu, khả năng cập nhật xu hướng, các chương trình ưu đãi tích hợp, trở thành người tiêu dùng thông minh qua việc xem các mẫu quảng cáo trên Facebook rất được người dùng quan tâm.

Xuất phát từ những kết quả thu được, tác giả đề xuất các hàm ý tham khảo cho các doanh nghiệp sau: giá trị thông tin là yếu tố có sức ảnh hưởng mạnh nhất đến nhận thức tránh né quảng cáo của người dùng Facebook trong khi giá trị giải trí có ít tác động hơn. Trên thực tế, bản thân người dùng đã có sã̃n những định kiến trong nhận thức đối với các quảng cáo và khi có thêm các căn cứ khác về khía cạnh thông tin (hữu ích, quan trọng, đa dạng, cập nhật, phù hợp) thì nhận thức này càng rõ nét. Tuy nhiên, về hành vi tránh né phát sinh thì giá trị thông tin lại có mức độ tác động thấp nhất trong tất cả các yếu tố của mô hình nghiên cứu đề xuất, sức ảnh hưởng lớn nhất rơi vào giá trị giải trí của quảng cáo.

Do vậy, để hạn hạn chế tối đa các hành vi loại bỏ, chặn, báo cáo sai phạm... đối với các quảng cáo trên Facebook, các doanh nghiệp nhất thiết phải tận dụng và khai thác tối đa giá trị giải trí trong các mẫu quảng cáo mà mình đưa ra. Khía cạnh này liên quan đến cách thức thể hiện thông điệp của hoạt động truyền thông sao cho phải tạo được cảm giác thích thú, dễ chịu, không gượng ép người dùng. Bên cạnh đó, để giảm thiểu đến mức thấp nhất các nhận thức tiêu cực, sự phớt lờ, không chú tâm của người xem... dẫn đến 
né tránh quảng cáo thì giá trị của thông tin là yếu tố cần lưu tâm. Để làm được điều đó, thông tin nhất thiết phải nhắm đúng đối tượng, đến đúng thời điểm và phù hợp với nhu cầu của người dùng.

Đối với giá trị khích lệ, đây là giá trị chỉ ảnh hưởng trực tiếp đến hành vi tránh né quảng cáo, doanh nghiệp nên hướng đến việc cung cấp những trải nghiệm tích cực, có sức động viên để thúc đẩy những hành vi tích cực của người dùng bằng các giải pháp thiết thực như: truyền thông theo xu hướng thị trường, cập nhật những trào lưu để tăng mức độ quan tâm, chia sẻ của người dùng. Khi đó, thông điệp quảng cáo sẽ được người xem chấp nhận thoải mái hơn thay vì tránh né.

\section{HẠN CHẾ VÀ HƯỚNG NGHIÊN CỬU TIẾP THEO}

Hạn chế chính của nghiên cứu chính là chưa xem xét các yếu tố liên quan đến môi trường, văn hóa, đặc tính cá nhân (độ tuổi, giới tính, trình độ học vấn, nghề nghiệp ...) có ảnh hưởng đến tránh né quảng cáo trên hai phương diện nhận thức và hành vi. Đồng thời, giới hạn nữa của đề tài chính việc chỉ chọn nghiên cứu quảng cáo trên mạng xã hội Facebook.

Vậy nên để kết quả được chuẩn xác hơn tác giả cũng đề xuất hướng nghiên cứu trong tương lai là kiểm định thêm những yếu tố thuộc về nhân khẩu tác động như thế nào đến hành vi tránh né quảng cáo cũng như có thể tiến xa hơn để nghiên cứu riêng cho từng loại website cụ thể như website công ty, cửa hàng trực tuyến, hay những trang mạng xã hội khác như Twitter, Intagram, Myspace, Zalo...

\section{TÀI LIỆ THAM KHẢO}

[1] D. S. Aaker and E. B. Donald, Causes of Irritation in Advertising, Journal of Marketing, vol. 49, no. 2, pp. 4757, 1985.

[2] L. K. Bracket and B.N. Carr, Cyberspace advertising vs. other media: Consumer vs. mature student attitudes (advertising attitudes), Journal of Advertising Research, vol. 41, no.5, pp. 23-32, 2001.

[3] C.H. Cho and H. J. Cheon, Why do people avoid advertising on the internet, Journal of Advertising, vol. 33, no.4, pp. 89-97, 2004.

[4] S. M. Choi, E. Kim, S. Kim and Y. H. Yeh, Factors Affecting Advertising Avoidance on Online Video Sites, The Journal of Advertising and Promotion Research, vol. 2, no. 1, pp. 87-121, 2013.

[5] M. Clancey, The television Audience Examined, Journal Advertising Research, vol. 39 no. 5, pp. 27-37, 1994.

[6] A.N. Diaz, K. Hammond and G. McWilliam, A Study of Web Use and Attitudes Amongst Novices, Moderate Users and Heavy Users, EMAC, pp. 1624-1635, 1997.

[7] R. H. Ducoffe, How Consumers Assess the Value of Advertising, Journal of Current Issues and Research in Advertising, vol. 17, no.1, pp. 1-18, 1995.

[8] R. H. Ducoffe, Advertising Value and Advertising on the Web, Journal of Advertising Research, vol. 36, no.5, pp. 21-35, 1996.

[9] S. M. Edwards, H. Li and J.H. Lee, Forced exposure and psychological reactance: Antecedents and consequences of the perceived intrusiveness of pup-up ads, Journal of Advertising, vol. 31, no.3, 83-95, 2002.

[10] J. Elisabeth, Network Television Streaming Technologies and the Shifting Television Social Sphere, Paper presented Media in Transition 6: Stone and Papyrus, Storage and Transmission, International Conference 2009 Massachusetts Institute of Technology, 2009.

[11] R. T. Hightower, Hulu: Video \& Effective Advertising, 2008.

[12] D. Hussain and H. Lasage, Online Video Advertisement Avoidance: Can Interactivity Help?, Journal of Applied Business Research, vol. 30, no. 1, 43-49, 2014. 
106 TÁC ĐộNG CỦA GIÁ TRI QUẢNG CÁO ĐẾN NHẬN THỨC VÀ HÀNH VI TRÁNH NÉ QUẢNG CÁO MỘT NGHIÊN CUUUU VỀ TRÁNH NÉ QUẢNG CÁO TRÊN FACEBOOK TẠI TP. HỒ CHÍ MINH

[13] S. Lee and J. R. Lumpkin, Differences in Attitude Toward TV Advertising: VCR Usage as a Moderator, International Journal of Advertising, vol. 11, no. 4, pp. 333-342, 1992.

[14] W. Li and Z. Huang, The Research of Influence Factors of Online Behavioral Advertising Avoidance, American Journal of Industrial and Business Management, vol. 6, pp. 947-957, 2016.

[15] K. Louise, G. Kerr and J. Drennan, Advoidance of Advertising in Social Networking Sites: The Teenage Perspective, Journal of Interactive Advertising, vol. 10, no. 2, pp. 16-27, 2010.

[16] T. Đ. Nguyễn và T. T. M. Nguyễn, Phương pháp nghiên cứu khoa học trong kinh doanh. Hà Nội. NXB Lao động Xã hội, 2012.

[17] Y. Pasadeos, Perceived Informativeness of and Irritation with Local Advertising, Journalism Quarterly, vol. 67, no. 1 , pp. 35-39, 1990.

[18] A. M. Rubin, M. P. Elizabeth and A. P. Robert, Loneliness, parasocial interaction, and local television news viewing, Human Communication Research, vol. 12, pp. 155-180, 1985.

[19] P. S. Speck and T. E. Micheal, Predictors of Advertising Advoidance in Print and Broadcast Media, Journal of Advertising, vol. 26, pp. 61-76, 1997.

[20] D. J. Xu, The influence of personalization in affecting consumer attitudes toward mobile advertising in China, The Journal of Computer Information Systems, vol. 47, no.2, pp. 9-19, 2007.

Ngày nhận bài: 07/09/2017

Ngày chấp nhận đăng: 05/01/2018 